\title{
Expression of diaphragmatic myostatin and correlation with apoptosis in rats with chronic obstructive pulmonary disease
}

\author{
$\mathrm{XU}_{\text {ZHOU }}{ }^{1}$, DAIJIAO $\mathrm{YI}^{2}$, YANHONG WU ${ }^{1}$, XINGHUA PEI ${ }^{1}$, \\ HAIMING YU ${ }^{1}$, YANHONG CHEN ${ }^{1}$, YU JIANG ${ }^{3}$ and WENPU $\mathrm{LI}^{2}$
}

\author{
Departments of ${ }^{1}$ Critical Care Medicine and ${ }^{2}$ Respiratory Medicine, Hunan Provincial People's Hospital; \\ ${ }^{3}$ Institute of Emergency Medicine, Hunan Provincial People's Hospital, Changsha, Hunan 410005, P.R. China
}

Received July 12, 2016; Accepted November 3, 2017

DOI: $10.3892 / \mathrm{etm} .2018 .5686$

\begin{abstract}
Chronic obstructive pulmonary disease (COPD) is characterized by progressive airflow limitation and loss of lung function. The present study aimed to investigate the diaphragmatic protein expression of myostatin and its correlation with apoptosis in a rat model of CPOD. Sprague Dawley rats were randomly divided into a control group and a COPD group, the latter of which were exposed to cigarette smoke to build a rat model of COPD. The validity of the COPD model was evaluated by assessment of lung function and histopathological analysis. Diaphragmatic myostatin expression and apoptosis were measured by western blot and terminal deoxynucleotidyl transferase deoxyuridine triphosphate nick end labeling, respectively. The rat model of COPD was efficiently established by cigarette smoke exposure. Diaphragmatic myostatin expression and apoptotic index in COPD rats were obviously increased as compared with that in the control animals. A positive correlation between diaphragmatic myostatin expression and apoptotic index was identified $(\mathrm{r}=0.857)$. Diaphragmatic myostatin overexpression in rats with COPD may promote diaphragmatic apoptosis and atrophy, leading to diaphragm weakness and respiratory muscle dysfunction, which is involved in the pathology of COPD.
\end{abstract}

\section{Introduction}

Chronic obstructive pulmonary disease (COPD) is one of the most common chronic illnesses and is now the third leading cause of death worldwide (1). COPD is characterized by progressive airflow limitation and loss of lung function. Four primary mechanisms, including oxidative stress, inflammation, protease-antiprotease imbalance and apoptosis, have

Correspondence to: Dr Wenpu Li, Department of Respiratory Medicine, Hunan Provincial People's Hospital, 61 Jiefang West Road, Changsha, Hunan 410005, P.R. China

E-mail: csliwenpu@163.com

Key words: chronic obstructive pulmonary disease, diaphragm, myostatin, apoptosis been implicated in the pathophysiological process of COPD $(2,3)$. The diaphragm is the principal muscle for breathing. In COPD patients, the diaphragm undergoes fiber type transformation, which results in diaphragmatic atrophy, injury and apoptosis (4). Morphological and functional changes of the diaphragm in COPD are complex, and the underlying mechanisms have remained to be fully elucidated. Myostatin, also known as growth differentiation factor 8 (GDF-8), is a negative regulator of skeletal muscle growth and is overexpressed in muscle wasting diseases. Myostatin knockout mice displayed an increase in muscle mass and myostatin transgenic mice developed cachexia characterized by extensive muscle loss (5). Under physiological conditions, myostatin mainly exists in the inactive form, and is activated and upregulated by hypoxia, acidosis and glucocorticoids (6,7). These risk factors for inducing myostatin expression are present in patients with COPD, particularly at moderate and severe disease stages. There is evidence that myostatin protein expression is significantly increased in COPD patients. However, whether myostatin is associated with diaphragmatic apoptosis and atrophy has remained elusive. In the present study, an experimental rat model of COPD induced by cigarette smoke exposure was generated to observe myostatin expression, diaphragmatic apoptosis and the correlation between them.

\section{Materials and methods}

Animals. Male Sprague Dawley rats (age, 8 weeks; weight, 200-250 g) were purchased from SJA Laboratory Animal Co., Ltd. (Changsha, China). The rats were housed in mesh cages with food and water supply under a 12 -h light/dark cycle for at least 5 days prior to the experiments. All procedures performed were approved by the Institutional Animal Ethics Committee and conformed to the guide for the care and use of experimental animals of Hunan Provincial People's Hospital (Changsha, China).

Cigarette smoke exposure. Cigarette smoke was generated from Furong Cigarettes (China Tobacco Hunan Industrial Co., Ltd, Changsha, China). The animals were randomly divided into two groups as follows: Rats undergoing sham exposure (control group) and rats undergoing cigarette smoke exposure (COPD group). Rats were exposed to mainstream smoke of 
cigarettes at a concentration of $300 \mathrm{mg}$ particulate matter $/ \mathrm{m}^{3}$ for $20 \mathrm{~min}$ for four times per day over 5 months (8).

Measurement of lung function. Lung function was assessed as previously described (9). In brief, the rats were anaesthetized with $10 \%$ chloral hydrate $(3 \mathrm{ml} / \mathrm{kg}$, corresponding to $300 \mathrm{mg} / \mathrm{kg}$ ). The trachea was opened with an inverted T-shaped incision between the 2nd and 3rd cartilage ring, rapidly intubated and the animal was placed into an HX200 respiratory-flow transducer (Xinhangxinye Co., Ltd., Beijing, China) for measuring the forced expiratory volume at $0.3 \mathrm{sec}$ and forced vital capacity $\left(\mathrm{FEV}_{0.3} / \mathrm{FVC}\right)$ and the peak expiratory flow (PEF). A total of $6 \mathrm{ml}$ air was injected at the end of the exhalation, which induced a passive deep inspiration, and the lung function was recorded.

Transmission electron microscopy (TEM) examination of diaphragms. Diaphragms were collected, weighed and fixed with $2.5 \%$ paraformaldehyde, and then refixed with $1 \%$ osmium tetroxide, washed with PBS, dehydrated with a graded ethanol series and pure acetone, paraffin-embedded, sectioned $(50 \mathrm{~nm})$, uranium-lead double stained (2\% uranyl acetate, saturated aqueous lead citrate) and observed under a H-7500 electron microscope (Hitachi, Tokyo, Japan).

Histopathological examination of the lung. The lung tissues were fixed in $4 \%$ paraformaldehyde for $12 \mathrm{~h}$, dehydrated using a graded ethanol series, and then placed in xylene for $2 \mathrm{~h}$, followed by paraffin embedding overnight. Sections ( $4 \mu \mathrm{m})$ were prepared and mounted on slides, and the samples were stained with H\&E for $10 \mathrm{~min}$ at room temperature and examined under an Olympus BX71 microscope (Olympus, Tokyo, Japan).

Mean linear intercept (MLI) and mean alveolar number (MAN) were examined as previously described (10). The MLI was used to estimate the average diameter of a single alveolus by using the formula MLI = total length/alveolar septal number. The intercepts of the alveolar septal number were counted at the intersection point of the two lines, and the total length of all of the lines combined divided by the number of intercepts provided the mean linear intercept for the region studied. The MAN was an indicator for the density of the alveoli, which was determined as the number of alveolar per square area in the field.

Assessment of diaphragmatic apoptosis. Diaphragmatic apoptosis was identified using the in situ terminal deoxynucleotidyl transferase deoxyuridine triphosphate nick end labeling (TUNEL) assay (KeyGen biotech, Nanjing, China) following the manufacturer's instructions. The diaphragmatic apoptotic index was the percentage of TUNEL-positive cells among the total cell population. Two slices of each sample were taken to acquire data from five different fields of view at high-power magnification (x400). At least 400 cells were examined.

Determination of myostatin protein. Western blot analysis was performed as previously described (11). In brief, the diaphragms were rinsed with ice-cold PBS, harvested with radioimmunoprecipitation assay buffer (Applygen, Beijing, China) and quantified using a bicinchoninic acid protein assay
Table I. Effects of COPD on the diaphragm mass, FEV0.3/FVC and FEP of rats.

\begin{tabular}{lcccc}
\hline Group & $\mathrm{n}$ & $\begin{array}{c}\text { Diaphragm } \\
\text { mass }(\mathrm{g})\end{array}$ & $\begin{array}{c}\mathrm{FEV}_{0.3} / \mathrm{FVC} \\
(\%)\end{array}$ & $\begin{array}{c}\mathrm{FEP} \\
(\mathrm{ml} / \mathrm{sec})\end{array}$ \\
\hline Control & 8 & $1.726 \pm 0.073$ & $83.5 \pm 4.9$ & $40.2 \pm 3.7$ \\
COPD & 8 & $1.311 \pm 0.156^{\mathrm{a}}$ & $66.2 \pm 4.1^{\mathrm{a}}$ & $24.8 \pm 2.2^{\mathrm{a}}$ \\
\hline
\end{tabular}

${ }^{\text {a }} \mathrm{P}<0.05$, as compared with control group. COPD, chronic obstructive pulmonary disease; $\mathrm{FEV}_{0.3} / \mathrm{FVC}$, forced vital capacity; $\mathrm{PEF}$, peak expiratory flow.

(WellBiology, Changsha, China). Total protein (30 $\mu \mathrm{g} / \mathrm{lane})$ was separated by $12 \%$ SDS-PAGE and transferred to polyvinylidene fluoride membranes (EMD Millipore, Billerica, MA, USA). The membranes were blocked in 5\% bovine serum albumin (Sigma-Aldrich; Merck KGaA, Darmstadt, Germany) for $1 \mathrm{~h}$ at room temperature and probed with antibody against myostatin (cat no. 19142-1-AP) and $\beta$-actin (cat no. 20536-1-AP; 1:1,000; Proteintech, Chicago, IL, USA) at $4^{\circ} \mathrm{C}$ overnight, followed by incubation with a secondary antibody (cat no. SA00001-2; 1:5,000; Proteintech, Chicago, IL, USA) conjugated to horseradish peroxidase at room temperature for $1 \mathrm{~h}$. Immunoreactivity was detected by enhanced chemiluminescent agent (Thermo Fisher Scientific, Inc., Waltham, MA, USA) according to the manufacturer's instructions. The protein expression levels were quantitatively analyzed and normalized against the $\beta$-actin loading control.

Statistical analysis. Values are expressed as the mean \pm standard deviation and analyzed using SPSS 20.0 (IBM Corp., Armonk, NY, USA). The two independent samples t-test was used for analysis of differences between the two groups. Pearson's linear correlation analysis was employed to identify any possible correlation between parameters. $\mathrm{P}<0.05$ was considered to indicate a statistically significant difference.

\section{Results}

COPD is associated with changes in diaphragm weight and lung function. The diaphragm mass, $\mathrm{FEV}_{0.3} / \mathrm{FVC}$ and $\mathrm{PEF}$ were determined at the end of the challenge procedure and the results are displayed in Table I. Compared with that in the control group, the diaphragm mass in the COPD group was significantly decreased $(1.726 \pm 0.073$ vs. $1.311 \pm 0.156 \mathrm{~g}$; $\mathrm{P}<0.05)$. The average values for $\mathrm{FEV}_{0.3} / \mathrm{FVC}$ and FEP were $83.5 \pm 4.9 \%$ and $40.2 \pm 3.7 \mathrm{ml} / \mathrm{sec}$ in the control group, and were significantly decreased to $66.2 \pm 4.1 \%$ and $24.8 \pm 2.2 \mathrm{ml} / \mathrm{sec}$ in the COPD group, respectively $(\mathrm{P}<0.05)$.

COPD is associated with changes of diaphragm ultrastructure and pulmonary histopathology. The lung tissues of COPD rats exhibited significant histopathological changes under light microscopy. Compared with those in the control group, the alveolar spaces were larger and the alveolar walls were thinner in the COPD group. Certain alveoli were broken 

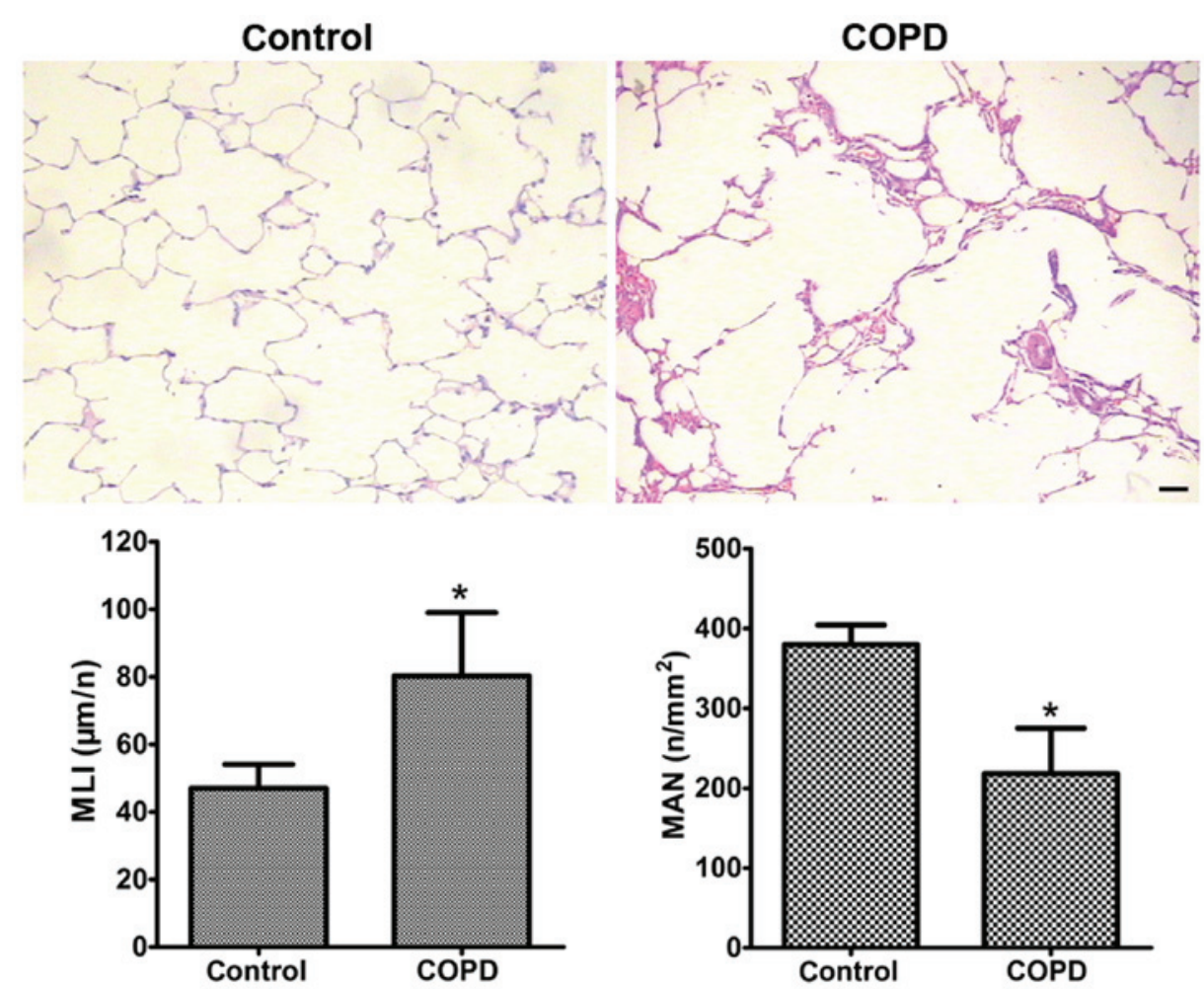

Figure 1. Effects of COPD on lung histopathology, MLI and MAN of rats. The lung tissues were stained with H\&E. COPD induced lung damage, as evidenced by inflammatory cell infiltration, exudate blockage of capillaries, as well as substantial interstitial cellular degeneration and necrosis. Representative images for each group are displayed, with each group containing 6-8 rats (scale bar, $50 \mu \mathrm{m}$ ). Values are expressed as the mean \pm standard deviation Two sections were randomly selected from the paraffin tissue samples of each animal. The sections were stained and then 5 fields of view in each slice were randomly selected for cell counts. " $\mathrm{P}<0.05$ when compared with the control group. COPD, chronic obstructive pulmonary disease; MLI, mean linear intercept; MAN, mean alveolar number.
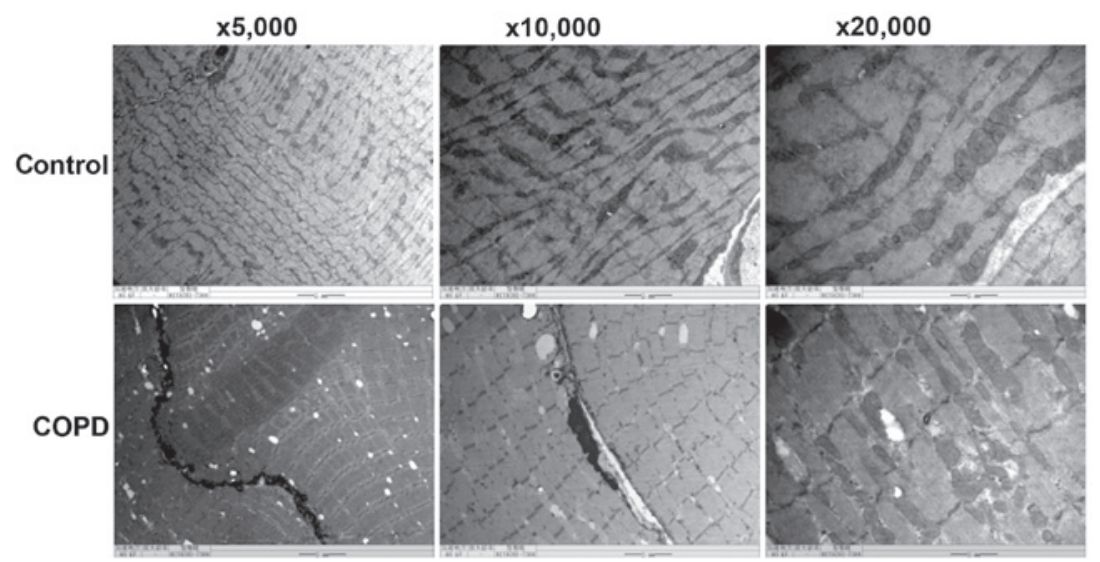

Figure 2. Effects of COPD on diaphragm ultrastructure of rats. COPD induced significant ultrastructural changes in the diaphragm, including muscle fiber atrophy, anomalies in sarcomere arrangement, myofilament breaking and dissolution, mitochondrial swelling and vacuolization, cristae lysis, karyopyknosis and nuclear chromatin aggregation and margination, as well as nuclear membrane thickening, shrinkage and irregularities (magnification, x5,000, x10,000 or x20,000). COPD, chronic obstructive pulmonary disease.

and had fused into bullae. In addition, infiltration of inflammatory cells into the mucosa, detachment of epithelial cells, submucosal gland hyperplasia and hypertrophy, as well as disorders and detachment of cilia were also observed in the COPD group (Fig. 1). The average MLI in the COPD group $(80.3 \pm 18.7 \mathrm{~mm})$ was $\sim 70 \%$ higher than that in the control group (47.0 $\pm 7.1 \mathrm{~mm} ; \mathrm{P}<0.05$; Fig. 1). The average MAN in the COPD group $\left(218.0 \pm 57.2 / \mathrm{mm}^{2}\right)$ was significantly lower than that in the control rats $\left(379.8 \pm 24.4 / \mathrm{mm}^{2}\right.$; P<0.05; Fig. 1). TEM indicated that compared with control rats, COPD rats exhibited obvious ultrastructural changes of the diaphragm, including muscle fiber atrophy, sarcomere arrangement anomaly, myofilament breakage and dissolution, mitochondrial swelling and vacuolization, cristae lysis, karyopyknosis and nuclear chromatin aggregation and margination, as well as nuclear membrane thickening, shrinkage and irregularities (Fig. 2).

COPD induces diaphragmatic apoptosis. TUNEL-positive diaphragm cells were identified as brown bodies by light 

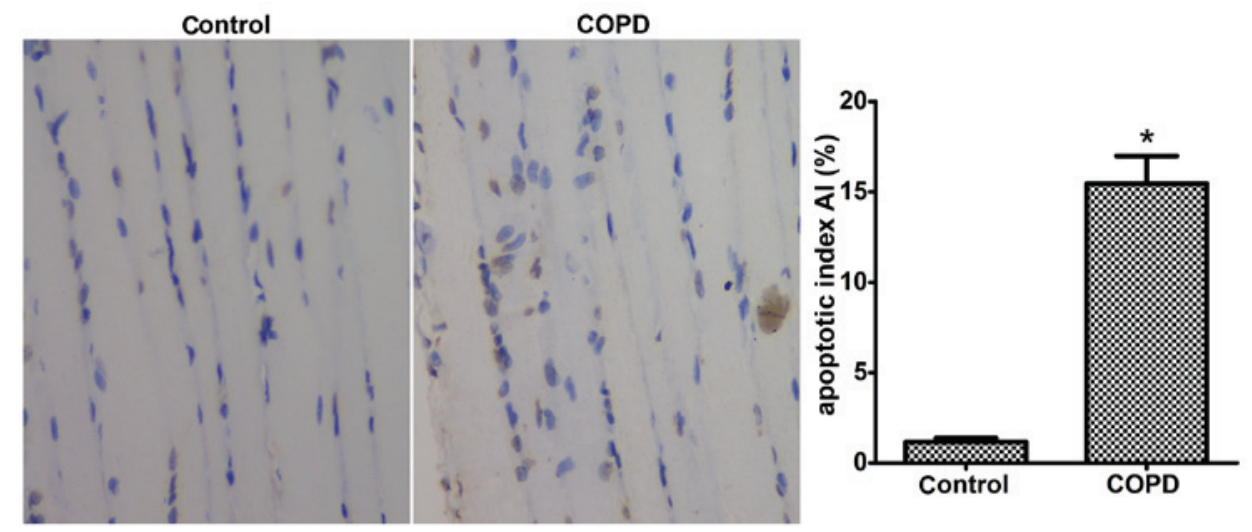

Figure 3. Diaphragmatic apoptosis in rats with COPD. COPD induced diaphragmatic apoptosis. Apoptosis was determined by terminal deoxynucleotidyl transferase deoxyuridine triphosphate nick end labeling. Representative images for each experimental group are displayed (magnification, $\mathrm{x} 400$ ), Values are expressed as the mean \pm standard deviation $(\mathrm{n} \geq 6)$. ${ }^{*} \mathrm{P}<0.05$ when compared with the control group. COPD, chronic obstructive pulmonary disease.

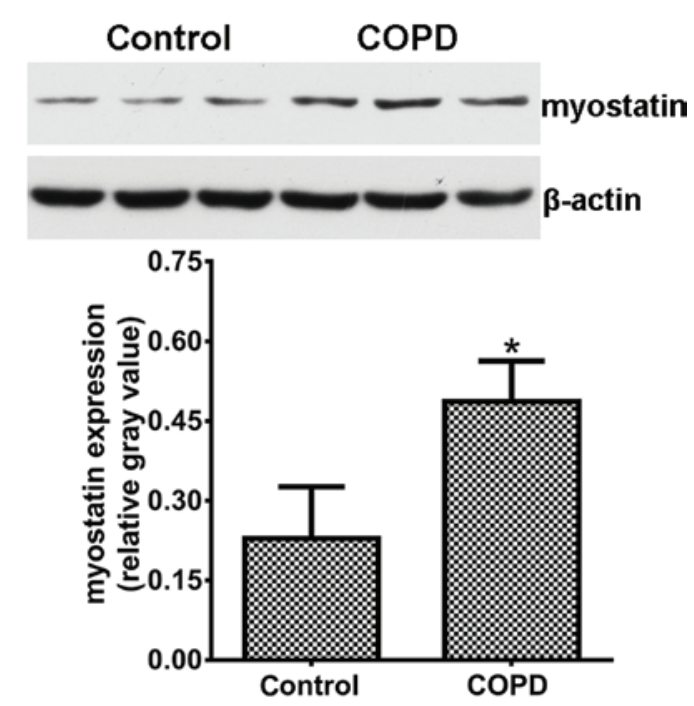

Figure 4. Diaphragmatic protein expression of myostatin in rats with COPD. COPD enhanced the diaphragmatic protein expression of myostatin. Myostatin protein expression was determined by western blot analysis and normalized to $\beta$-actin. Values are expressed as the mean \pm standard deviation $(\mathrm{n} \geq 6)$. ${ }^{*} \mathrm{P}<0.05$ when compared with the control group. COPD, chronic obstructive pulmonary disease.

microscopic analysis. The results indicated that the amount of TUNEL-positive cells was significantly greater in the diaphragms of COPD rats compared with that in control rats. The apoptotic index was $15.46 \pm 1.53 \%$ in the COPD group, which was significantly higher than that in the control group (1.17 $\pm 0.21 \%$; P<0.05; Fig. 3).

Myostatin expression is upregulated in the diaphragms of $C O P D$ rats. Myostatin expression in the diaphragms was assessed by western blot analysis and the results are displayed in Fig. 4. The expression levels of myostatin in the diaphragms of COPD rats were obviously increased as compared with those in the control animals $(\mathrm{P}<0.05)$.

Myostatin expression is correlated with diaphragmatic degeneration in COPD rats. A positive correlation was identified between the levels of myostatin and the diaphragmatic apoptotic index $(\mathrm{r}=0.865 ; \mathrm{P}<0.01)$. In addition, a negative correlation was identified between the levels of myostatin and the diaphragm mass ( $r=-0.777$; $<<0.05$; Fig. 5).

\section{Discussion}

COPD is characterized by progressive and incomplete reversible progressive airflow limitation, which remains a challenge for clinicians and represents a global health burden. Smoking is one of the most important risk factors for COPD, as harmful gases or particles in cigarette smoke may induce abnormal pulmonary inflammation (12). In the present study, an animal model of COPD was established through a modified protocol comprising a 5-month challenge of the rats through exposure to cigarette smoke (13). The presence of airflow obstruction is key in the diagnosis of COPD, and the present results revealed that this rat model was associated with a significant decrease in FEV0.3/FVC and FEP, which are critical parameters of lung injury in obstructive lung disease (14). In addition, the histopathological changes of lungs and diaphragms of COPD rats were similar to those seen in humans with COPD. In support of the pathological results, the MLI and MAN in the COPD model group were measured for quantitative analysis of lung structure and function (15). The results indicated enlargement of air spaces with a significant decrease in the alveolar number in the COPD rats as compared with those in the control, which provided direct evidence of lung injury. MLI and MAN are accurate and efficient indicators to reflect alveolar airspace size and lung architecture, and these parameters reasonably demonstrated that cigarette smoke exposure was an efficient way to establish a COPD model in rats.

Respiratory muscle dysfunction and particularly diaphragm dysfunction may induce respiratory failure, which was reported to be the leading cause of death in patients with COPD (16). Assessment of the pathogenesis of COPD has led to the recognition of apoptosis as an important factor in clinical COPD and experimental models thereof (17). Apoptosis may be triggered through three major pathways, one of which involves death receptor ligation, another is based on the release of cytochrome $c$ from mitochondria, and the third is the endoplasmic reticulum pathway (18). Skeletal muscle cell apoptosis may be responsible for muscle atrophy, which induces weight 

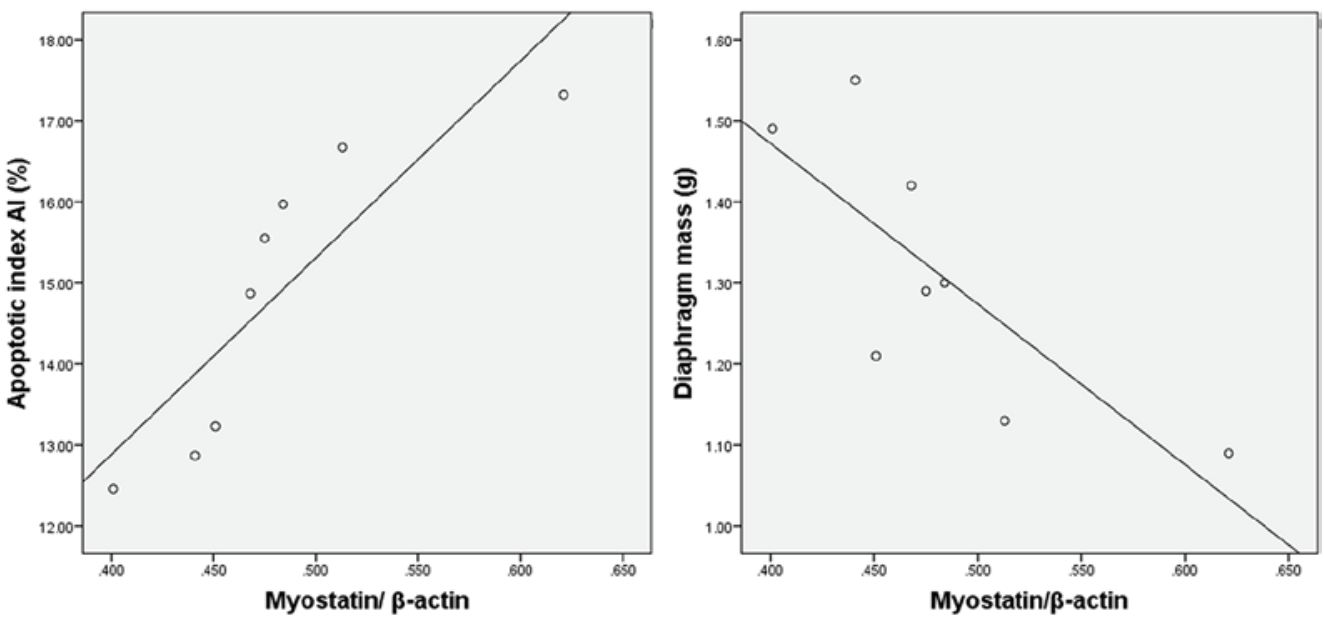

Figure 5. Correlation between diaphragmatic myostatin expression and apoptosis or diaphragm mass in rats with COPD. COPD, chronic obstructive pulmonary disease. A positive correlation was identified between myostatin expression and diaphragmatic apoptotic index $(\mathrm{r}=0.865 ; \mathrm{P}=0.006)$ and a negative correlation was identified between myostatin expression and the diaphragm mass $(\mathrm{r}=-0.777 ; \mathrm{P}=0.023)$.

loss and weakness in patients with COPD and seriously affects their quality of life. As the pivotal respiratory muscle, diaphragmatic apoptosis has important roles in the pathogenesis of COPD. The present results indicated a significant loss of diaphragm mass in COPD rats, which indicated diaphragmatic atrophy. Furthermore, the apoptotic index was significantly increased in the diaphragms of COPD rats. The present results therefore indicated diaphragmatic apoptosis in COPD, which is consistent with those of previous studies $(18,19)$. However, the exact mechanisms of diaphragmatic apoptosis and their role in the pathophysiological processes of COPD remain to be fully elucidated. The Fas/Fas ligand pathway was reported to participate in the regulation of diaphragmatic apoptosis (20), and diaphragmatic fiber type transformation (21). Diaphragms of COPD patients generated a lower specific force, and Type I fibers generated a lower specific force than Type II fibers (22). Fiber type transformations in COPD decreased diaphragmatic force generation and finally resulted in respiratory failure (23). Preventing diaphragmatic apoptosis and reducing fiber type transformation may be promising approaches for preventing and curing respiratory failure in COPD.

Myostatin, also known as GDF-8, is a member of the TGF- $\beta$ superfamily that is highly expressed in skeletal muscle (24), functions as a negative regulator of skeletal muscle growth and is overexpressed in muscle atrophy and wasting diseases, including bedrest and disuse atrophy, anorexia nervosa and cancer-associated cachexia. Myostatin expression limits the size of muscles during development (25). Myostatin-null mice exhibited muscle hypertrophy, and myostatin transgenic mice displayed muscle atrophy and cachexia. Myostatin protein remains inactive until it is cleaved by a protease. Activated myostatin binds to the activin type II receptor, recruits activin receptor-like kinase 4 and then initiates cell signaling cascades in the muscle, which include the SMAD and mitogen-activated protein kinase families (26). Furthermore, to cause muscle hypertrophy, AKT kinase may be inhibited by myostatin through partially suppressing protein synthesis (27). In the present study, myostatin protein expression was assessed by western blot analysis, indicating that diaphragmatic myostatin in rats with COPD was significantly overexpressed compared with that in the control rats, which was consistent with the results of previous studies $(6,28)$.

After diaphragm mass, apoptosis and myostatin expression were assessed in COPD rats, their correlation was then assessed in the present study. A negative correlation between myostatin expression and diaphragm mass, and a positive correlation between myostatin expression and apoptosis was identified in the diaphragms of COPD rats. However, the underlying mechanisms of the link between these parameters remains to be fully elucidated and requires to be further investigated. It may be speculated that myostatin overexpression in the diaphragm promotes diaphragmatic apoptosis and atrophy, leading to diaphragm weakness and respiratory muscle dysfunction, and finally aggravates the progression of COPD. Type II respiratory failure and persistent $\mathrm{CO}_{2}$ retention are present in the late stage of COPD, are closely associated with respiratory muscle dysfunction and are the major cause of COPD-associated death (29). Inhibition of myostatin in the early stage of COPD may reduce diaphragmatic apoptosis and improve respiratory muscle function, which may provide a promising strategy for the prevention and treatment of COPD.

In conclusion, a rat model of COPD was established using chronic cigarette smoke exposure. A positive correlation was identified between myostatin expression and diaphragmatic apoptosis. Myostatin may promote diaphragmatic apoptosis and atrophy, cause respiratory muscle dysfunction and contribute to the pathogenesis of COPD. However, the present study had certain limitations. No method to interfere with the protein expression of myostatin was employed to then observe its potential effect to reduce the severity of COPD and diaphragmatic apoptosis. A future study by our group will assess whether myostatin regulates apoptosis in COPD. Identification of the role of myostatin in COPD may provide novel potential targets for the prevention or cure of COPD.

\section{Acknowledgements}

This study was financially supported by a Program of the Hunan Provincial Department of Science and Technology (grant no. 2010SK3071). 


\section{References}

1. Lozano R, Naghavi M, Foreman K, Lim S, Shibuya K, Aboyans V, Abraham J, Adair T, Aggarwal R, Ahn SY, et al: Global and regional mortality from 235 causes of death for 20 age groups in 1990 and 2010: A systematic analysis for the Global Burden of Disease Study 2010. LANCET 380: 2095-2128, 2012.

2. Barnes PJ: Cellular and molecular mechanisms of chronic obstructive pulmonary disease. Clin Chest Med 35: 71-86, 2014.

3. Hillas G, Nikolakopoulou S, Hussain S and Vassilakopoulos T: Antioxidants and mucolytics in COPD management: When (if ever) and in whom? Curr Drug Targets 14: 225-234, 2013.

4. Ottenheijm CA, Heunks LM and Dekhuijzen RP: Diaphragm adaptations in patients with COPD. Respir Res 9: 12, 2008.

5. Elkasrawy MN and Hamrick MW: Myostatin (GDF-8) as a key factor linking muscle mass and bone structure. J Musculoskelet Neuronal Interact 10: 56-63, 2010.

6. Hayot M, Rodriguez J, Vernus B, Carnac G, Jean E, Allen D, Goret L, Obert P, Candau R and Bonnieu A: Myostatin up-regulation is associated with the skeletal muscle response to hypoxic stimuli. Mol Cell Endocrinol 332: 38-47, 2011.

7. Ma K, Mallidis C, Bhasin S, MahabadiV, Artaza J, Gonzalez-Cadavid N, Arias J and Salehian B: Glucocorticoid-induced skeletal muscle atrophy is associated with upregulation of myostatin gene expression. Am J Physiol Endocrinol Metab 285: E363-E371, 2003.

8. Vlahos R and Bozinovski S: Recent advances in pre-clinical mouse models of COPD. Clin Sci (Lond) 126: 253-265, 2014.

9. Wang Y, Jiang X, Zhang L, Wang L, Li Z and Sun W: Simvastatin mitigates functional and structural impairment of lung and right ventricle in a rat model of cigarette smoke-induced COPD. Int J Clin Exp Pathol 7: 8553-8562, 2014.

10. Wang Y, Jiang X, Zhang L, Wang L, Li Z and Sun W: Simvastatin mitigates functional and structural impairment of lung and right ventricle in a rat model of cigarette smoke-induced COPD. Int J Clin Exp Pathol 7: 8553-8562, 2014.

11. Jiang Y, Gao M, Wang W, Lang Y, Tong Z, Wang K, Zhang H, Chen G, Liu M, Yao Y and Xiao X: Sinomenine hydrochloride protects against polymicrobial sepsis via autophagy. Int J Mol Sci 16: 2559-2573, 2015.

12. Margaritopoulos GA, Vasarmidi E, Jacob J, Wells AU and Antoniou KM: Smoking and interstitial lung diseases. Eur Respir Rev 24: 428-435, 2015.

13. Fricker M, Deane A and Hansbro PM: Animal models of chronic obstructive pulmonary disease. Expert Opin Drug Discov 9: 629-645, 2014

14. Morris ZQ, Coz A and Starosta D: An isolated reduction of the FEV3/FVC ratio is an indicator of mild lung injury. Chest 144: 1117-1123, 2013

15. Andersen MP, Parham AR, Waldrep JC, McKenzie WN and Dhand R: Alveolar fractal box dimension inversely correlates with mean linear intercept in mice with elastase-induced emphysema. Int J Chron Obstruct Pulmon Dis 7: 235-243, 2012.
16. Wouters EF: Local and systemic inflammation in chronic obstructive pulmonary disease. Proc Am Thorac Soc 2: 26-33, 2005.

17. Plataki M,Tzortzaki E, Rytila P,Demosthenes M,Koutsopoulos A and Siafakas NM: Apoptotic mechanisms in the pathogenesis of COPD. Int J Chron Obstruct Pulmon Dis 1: 161-171, 2006.

18. Degens H, Swisher AK, Heijdra YF, Siu PM, Dekhuijzen PN and Alway SE: Apoptosis and Id2 expression in diaphragm and soleus muscle from the emphysematous hamster. Am J Physiol Regul Integr Comp Physiol 293: R135-R144, 2007.

19. Barreiro E, Ferrer D, Sanchez F, Minguella J, Marin-Corral J, Martinez-Llorens J, Lloreta J and Gea J: Inflammatory cells and apoptosis in respiratory and limb muscles of patients with COPD. J Appl Physiol (1985) 111: 808-817, 2011.

20. Tan Y, Sun L, Gao J, and Ouyang X: Apoptosis of diaphragmatic muscle cell in Chronic obstructive pulmonary disease. J Clin Pulm Med, 15: 1580-1582, 2010.

21. Ji L, Sun L, Tan Y and Gu W: Adaptations of diaphragmatic muscle fibres and apoptosis of diaphragmatic muscle cell in rats with chronic obstructive pulmonary disease. Acta U Med Nanjing(Natural Science) 32: 194-198, 2012.

22. Levine S, Nguyen T, Kaiser LR, Rubinstein NA, Maislin G, Gregory C, Rome LC, Dudley GA, Sieck GC and Shrager JB: Human diaphragm remodeling associated with chronic obstructive pulmonary disease: Clinical implications. Am J Respir Crit Care Med 168: 706-713, 2003.

23. Sauleda RJ: Clinical consequences of muscle dysfunction in chronic obstructive pulmonary disease. Nutr Hosp 21 (Suppl 3): 69-75, 2006 (In Spanish).

24. McPherron AC, Lawler AM and Lee SJ: Regulation of skeletal muscle mass in mice by a new TGF-beta superfamily member. Nature 387: 83-90, 1997.

25. Amthor H, Huang R, McKinnell I, Christ B, Kambadur R, Sharma $\mathrm{M}$ and Patel K: The regulation and action of myostatin as a negative regulator of muscle development during avian embryogenesis. Dev Biol 251: 241-257, 2002.

26. Yang W, Chen Y, Zhang Y, Wang X, Yang N and Zhu D: Extracellular signal-regulated kinase $1 / 2$ mitogen-activated protein kinase pathway is involved in myostatin-regulated differentiation repression. Cancer Res 66: 1320-1326, 2006.

27. Sartori R, Gregorevic P and Sandri M: TGFbeta and BMP signaling in skeletal muscle: potential significance for muscle-related disease. Trends Endocrinol Metab 25: 464-471, 2014.

28. Testelmans D, Crul T, Maes K, Agten A, Crombach M, Decramer M and Gayan-Ramirez G: Atrophy and hypertrophy signalling in the diaphragm of patients with COPD. Eur Respir J 35: 549-556, 2010.

29. Gea J, Agusti A and Roca J: Pathophysiology of muscle dysfunction in COPD. J Appl Physiol (1985) 114: 1222-1234, 2013.

This work is licensed under a Creative Commons Attribution-NonCommercial-NoDerivatives 4.0 International (CC BY-NC-ND 4.0) License. 